NEUROLOGICAL PICTURE

\title{
Pulmonary apex schwannoma
}

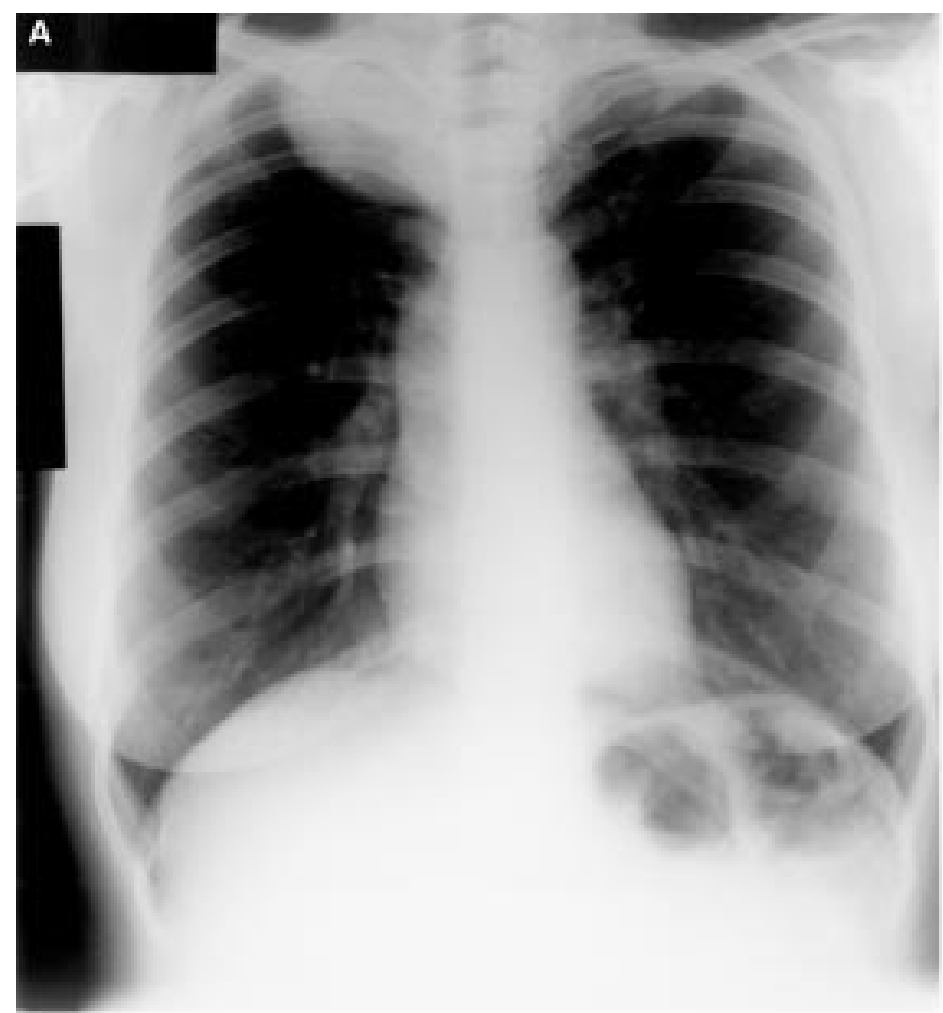

Chest radiography $(A)$ and thoracic $M R I(B)$ of the patient.

A 45 year old previously fit woman presented with a six month history of fatigue, generalised arthralgia, and burning discomfort in both legs. General examination was unremarkable except for a dull percussion note at the right lung apex. Neurological assessment showed mild spastic paraparesis with absent abdominal reflexes and reduced pain and temperature sensations below the fourth thoracic level. Extensive haematological, biochemical, immunological, serological, and electrophysiological investigations did not show any

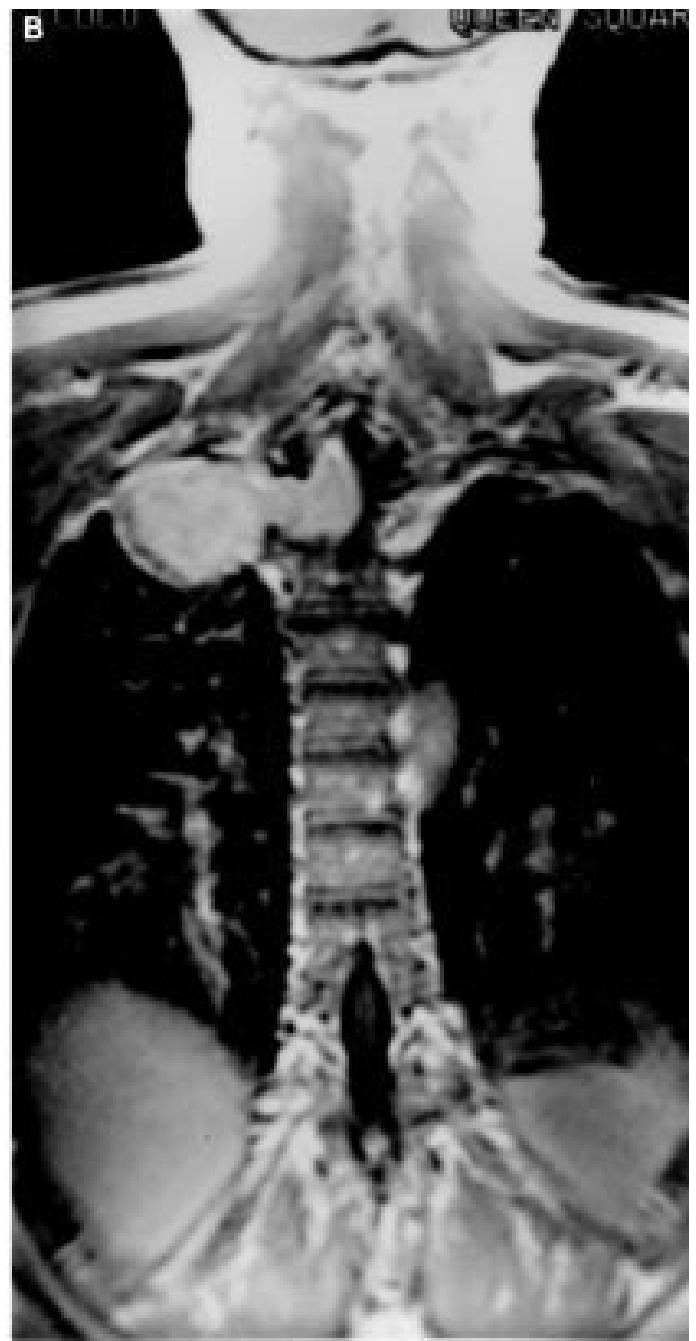
abnormalities. Chest radiography, however, disclosed an opacity at the right pulmonary apex (figure, A). Magnetic resonance imaging confirmed this (figure, B). The mass extended through the right neural foramen at the second thoracic level and caused compression of the spinal cord. These radiological features were suggestive of a dumbbell neurofibroma. The lesion was excised via a cervicothoracic approach. The histology was confirmed as a schwannoma. Postoperatively her symptoms resolved. Retrospectively, detailed family history was negative for neurofibromatosis.

KEYOUMARS ASHKAN ADRIAN T H CASEY Department of Surgical Neurology, National Hospital for Neurology and Neurosurgery, Queen Square, London WC1N 3BG. 